\title{
A Cell-Based Smoothed XFEM for Fracture in Piezoelectric Materials
}

\author{
Li Ming Zhou, Guang Wei Meng, Feng Li, and Shuai Gu
}

School of Mechanical Science and Engineering, Jilin University, Changchun 130025, China

Correspondence should be addressed to Shuai Gu; 444934246@qq.com

Received 29 July 2015; Revised 23 November 2015; Accepted 9 December 2015

Academic Editor: Bert Blocken

Copyright (C) 2016 Li Ming Zhou et al. This is an open access article distributed under the Creative Commons Attribution License, which permits unrestricted use, distribution, and reproduction in any medium, provided the original work is properly cited.

This paper presents a cell-based smoothed extended finite element method (CS-XFEM) to analyze fractures in piezoelectric materials. The method, which combines the cell-based smoothed finite element method (CS-FEM) and the extended finite element method (XFEM), shows advantages of both methods. The crack tip enrichment functions are specially derived to represent the characteristics of the displacement field and electric field around the crack tip in piezoelectric materials. With the help of the smoothing technique, integrating the singular derivatives of the crack tip enrichment functions is avoided by transforming interior integration into boundary integration. This is a significant advantage over XFEM. Numerical examples are presented to highlight the accuracy of the proposed CS-XFEM with the analytical solutions and the XFEM results.

\section{Introduction}

Because of their inherent coupling of electric and mechanical behaviors, piezoelectric materials have been widely used in sensors, actuators, signal transmitters and surface acoustic wave devices, aerospace panels, and civil structures. In those applications, piezoelectric materials may experience high mechanical stresses and electric field concentrations. As a result they may fail due to dielectric breakdown or fractures. These materials are usually inhomogeneous and brittle, with low ultimate tensile strength and fracture toughness. Therefore, defects such as cracks and voids should be detected to ensure reliability and durability of the piezoelectric structures. Numerical simulation of fractures in piezoelectric ceramics was conducted in [1], using finite element method (FEM) $[2,3]$, boundary element method (BEM) $[4,5]$, meshless method [6], and extended finite element method (XFEM) [7-9].

The theoretical fundamentals of piezoelectric fracture mechanics for cracks were presented in $[10,11]$. The analytical work to investigate the fracture mechanics of piezoelectric structures was based on Lekhnitskii and Stroh formalism [12]. An elliptic hole with a major axis perpendicular to the polarization direction inside piezoelectric ceramic and the field variables around the cavity were studied in [13].
An overview and critical discussion about the present state in the field of piezoelectric fracture mechanics were given in [14]. A survey on using numerical methods of crack analyses in piezoelectric medium along with FEM to solve fracture parameters is presented in [15]. Recently, XFEM was applied to analyze the $2 \mathrm{D}$ crack problems and fully coupled piezoelectric effect of piezoelectric ceramics [16]. The $\mathbf{M}$ integral and $\mathbf{J}$ integral were used to solve the stress intensity factors and electric displacements intensity factors $[17,18]$. The newly developed crack tip enrichment functions of XFEM were found suitable for cracks in piezoelectric materials [19]. An extension of XFEM for dynamic fracture in piezoelectric materials was presented in [20].

In recent years, the smoothed FEM has been well adopted to solve fracture mechanics problem [21-27]. Based on generalized gradient smoothing technique a lot of novel and powerful numerical methods have been developed [2836]. A cell-based smoothed finite element method (CSFEM) has been developed with the smoothing domains constructed based on cell of the elements. In the method line integration was used along the boundaries of the smoothing cells instead of area integration. Moreover, CS-FEM does not need mapping and derivatives. It also showed that the results are less sensitive to distorted elements. 
In terms of the advantages, smoothing technique was incorporated into the extended FEM [37-39]. An edge-based smoothed XFEM was developed to combine the advantages of the edge-based smoothed FEM and the XFEM [40]. A node-based smoothed XFEM was applied to linear elastic fracture mechanics [41].

Recently, extended finite element method, collocation boundary element, cell-based smoothed finite element method, and so forth were used to solve the problem of fracture in piezoelectric materials. The extended finite element method focuses on the definition of new enrichment functions suitable for cracks in piezoelectric structures and generalized domain integrals are used for the determination of crack tip parameters [42]. The collocation boundary element with subdomain technique is developed, whereby the fundamental solutions are computed by a fast numerical algorithm applying Fourier series [43]. The cell-based smoothed finite element method and VCCT have been used to simulate the fracture mechanics of piezoelectric materials. A piezoelectric element tailored for VCCT was used to study the crack of piezoelectric materials. CS-FEM and VCCT were introduced into fracture mechanics of piezoelectric materials and CSFEM-VCCT for piezoelectric material with cracks was put forward [44]. In this paper cell-based smoothed XFEM (CS-XFEM) is extended to simulate flaws in piezoelectric structures. CS-XFEM combining characteristics of extended finite element method and smoothed finite element method can improve the accuracy of XFEM. Nodal enrichment can model crack propagation without remeshing. CS-XFEM has advantages that the crack tip element does not need fine division; the shape function is simple and no derivatives of shape functions are needed.

This paper is outlined as follows. In Section 2, the governing equations of piezoelectric materials are introduced. Section 3 focuses on the formulation of cell-based smoothed extended finite element method. Section 4 presents the electromechanical $J$-integral for 2D crack analysis. In Section 5, numerical examples with the assumption of impermeable crack face boundary conditions are presented to demonstrate the accuracy and efficiency of CS-XFEM. Section 6 is the conclusion.

\section{Governing Equations}

The electroelastic response of a piezoelectric body of volume $\Omega$ and regular boundary surface $S$ is governed by the mechanical and electrostatic equilibrium equations:

$$
\begin{gathered}
\sigma_{i j, j}+f_{i}=0 \quad \text { in } \Omega, \\
D_{i, i}-t=0 \quad \text { in } \Omega,
\end{gathered}
$$

where $f_{i}$ is mechanical body force, $t$ is electric body charge, $\sigma_{i j}$ is the symmetric Cauchy stress tensor, and $D_{i}$ is electric displacement vector components.

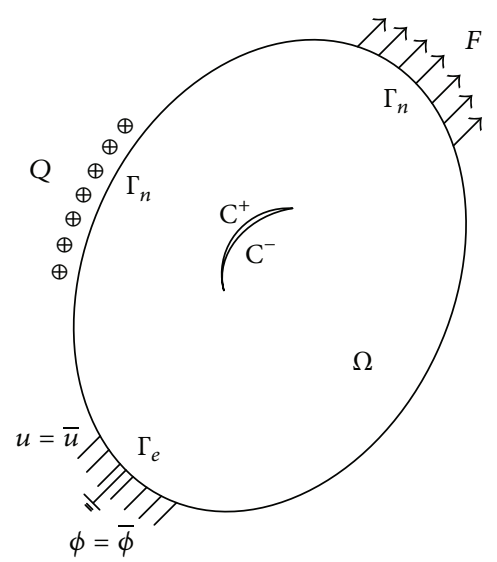

Figure 1: Piezoelectric domain with a crack.

The constitutive equations for a two-dimensional piezoelectric material in the $x-z$ plane can be expressed in terms of the stress and the electric field:

$$
\begin{aligned}
& \sigma_{i j}=C_{i j k l} \varepsilon_{k l}-e_{k i j} E_{k}, \\
& D_{i}=e_{i k l} \varepsilon_{k l}+\kappa_{i k} E_{k},
\end{aligned}
$$

where $\varepsilon_{k l}, D_{i}$, and $E_{k}$ are the strain tensor, the electric displacement vector, and the electric field vector, respectively; $C_{i j k l}, e_{k i j}$, and $\kappa_{i k}$ denote elastic stiffness at constant electric field, piezoelectric constants, and dielectric permittivity at constant strain, respectively.

The strains tensor are related to displacements by

$$
\varepsilon_{i j}=\frac{\left(u_{i, j}+u_{j, i}\right)}{2}
$$

and the electric field vector is related to electric potential by

$$
E_{i}=-\phi_{, i} .
$$

The piezoelectric body $\Omega$ could be subjected to the following essential and natural boundary conditions (Figure 1).

Essential Boundary Conditions. Consider

$$
\begin{aligned}
u & =\bar{u} \\
\text { or } \phi & =\bar{\phi}
\end{aligned}
$$

on $\Gamma_{e}$

Natural Boundary Conditions. Consider

$$
\begin{aligned}
\sigma_{i j} n_{j} & =\mathbf{f}_{i} \\
\text { (or) } D_{i} n_{i} & =-\mathbf{g} \\
& \text { on } \Gamma_{n},
\end{aligned}
$$

where $\bar{u}, \bar{\phi}, \mathbf{f}_{i}, \mathbf{g}$, and $n_{i}$ are mechanical displacement, electric potential, surface force components, surface charge, and 
outward unit normal vector components, respectively. The crack faces $\mathrm{C}^{+}$and $\mathrm{C}^{-}$are considered traction-free. The cracks are assumed to be electrically impermeable. Nevertheless, extension to limited permeable cracks is possible.

The two-dimensional matrix form of the mechanical and electrical constitutive equations can be given by [15]

$$
\begin{gathered}
{\left[\begin{array}{c}
\sigma_{x} \\
\sigma_{z} \\
\tau_{x z}
\end{array}\right]=\left[\begin{array}{ccc}
\mathbf{C}_{11} & \mathbf{C}_{13} & 0 \\
\mathbf{C}_{31} & \mathbf{C}_{33} & 0 \\
0 & 0 & \mathbf{C}_{55}
\end{array}\right]\left[\begin{array}{c}
\varepsilon_{x} \\
\varepsilon_{z} \\
\gamma_{x z}
\end{array}\right]-\left[\begin{array}{cc}
0 & \mathbf{e}_{31} \\
0 & \mathbf{e}_{33} \\
\mathbf{e}_{15} & 0
\end{array}\right]\left[\begin{array}{l}
E_{x} \\
E_{z}
\end{array}\right],} \\
{\left[\begin{array}{c}
D_{x} \\
D_{z}
\end{array}\right]=\left[\begin{array}{ccc}
0 & 0 & \mathbf{e}_{15} \\
\mathbf{e}_{31} & \mathbf{e}_{33} & 0
\end{array}\right]\left[\begin{array}{c}
\varepsilon_{x} \\
\varepsilon_{z} \\
\gamma_{x z}
\end{array}\right]+\left[\begin{array}{cc}
\boldsymbol{\kappa}_{11} & 0 \\
0 & \boldsymbol{\kappa}_{33}
\end{array}\right]\left[\begin{array}{l}
E_{x} \\
E_{z}
\end{array}\right],}
\end{gathered}
$$

where $\mathbf{C}_{i j}$ are the elastic compliance constants, $\mathbf{e}_{i j}$ are piezoelectric constants, and $\boldsymbol{\kappa}_{i i}$ are the dielectric constants.

\section{Cell-Based Smoothed Extended Finite Element Method}

In CS-XFEM, the approximation of displacement and electric potential field in a piezoelectric material are given by

$$
\begin{aligned}
& \mathbf{u}^{h}(\mathbf{x})=\sum_{I \in N^{\mathrm{CS}-\mathrm{FEM}}} N_{I}^{u}(\mathbf{x}) \mathbf{u}_{I} \\
& +\sum_{J \in N^{\text {CS }-c}} N_{J}^{u}(\mathbf{x})\left(H(\mathbf{x})-H\left(\mathbf{x}_{J}\right)\right) \mathbf{a}_{J} \\
& +\sum_{K \in N^{\text {CS. } f}} N_{K}^{l}(\mathbf{x}) \sum_{l=1}^{4}\left(\mathbf{F}_{l}(\mathbf{x})-\mathbf{F}_{l}\left(\mathbf{x}_{K}\right)\right) \mathbf{b}_{K}^{l}, \\
& \boldsymbol{\Phi}^{h}(\mathbf{x})=\sum_{I \in N^{\mathrm{CS}-\mathrm{FEM}}} N_{I}^{\phi}(\mathbf{x}) \boldsymbol{\Phi}_{I} \\
& +\sum_{J \in N^{\mathrm{CS}-c}} N_{J}^{\phi}(\mathbf{x})\left(H(\mathbf{x})-H\left(\mathbf{x}_{J}\right)\right) \boldsymbol{\alpha}_{J} \\
& +\sum_{K \in N^{\mathrm{cS}-f}} N_{K}^{\phi}(\mathbf{x}) \sum_{l=1}^{4}\left(\mathbf{F}_{l}(\mathbf{x})-\mathbf{F}_{l}\left(\mathbf{x}_{K}\right)\right) \boldsymbol{\beta}_{K}^{l},
\end{aligned}
$$

where $N_{I}^{u}(\mathbf{x}), N_{J}^{u}(\mathbf{x})$, and $N_{K}^{l}(\mathbf{x})$ are shape functions of the nodal displacement, while $\mathbf{u}_{I}$ are the nodal degrees of freedom associated with node $I$, and $\mathbf{a}_{J}$ and $\mathbf{b}_{K}$ are additional nodal degrees of freedom corresponding to the Heaviside function $H(\mathbf{x})$ and the near-tip functions, $\left\{\mathbf{F}_{l}\right\}_{1 \leq l \leq 4}$, respectively. $N_{I}^{\phi}(\mathbf{x}), N_{J}^{\phi}(\mathbf{x})$, and $N_{K}^{\phi}(\mathbf{x})$ are shape functions of the nodal electric potential, while $\Phi_{I}$ are the nodal degrees of freedom associated with node $I$, and $\boldsymbol{\alpha}_{I}$ and $\boldsymbol{\beta}_{K}$ are additional nodal degrees of freedom corresponding to the Heaviside function $H(\mathbf{x})$ and the near-tip functions, $\left\{\mathbf{F}_{l}\right\}_{1 \leq l \leq 4}$, respectively.

Nodes in set $N^{\text {CS-c }}$ have supports split by crack and nodes in set $N^{\mathrm{CS}-f}$ which belong to the smoothing domains contain a crack tip. These nodes are enriched with the Heaviside and asymptotic branch function fields depicted with squares

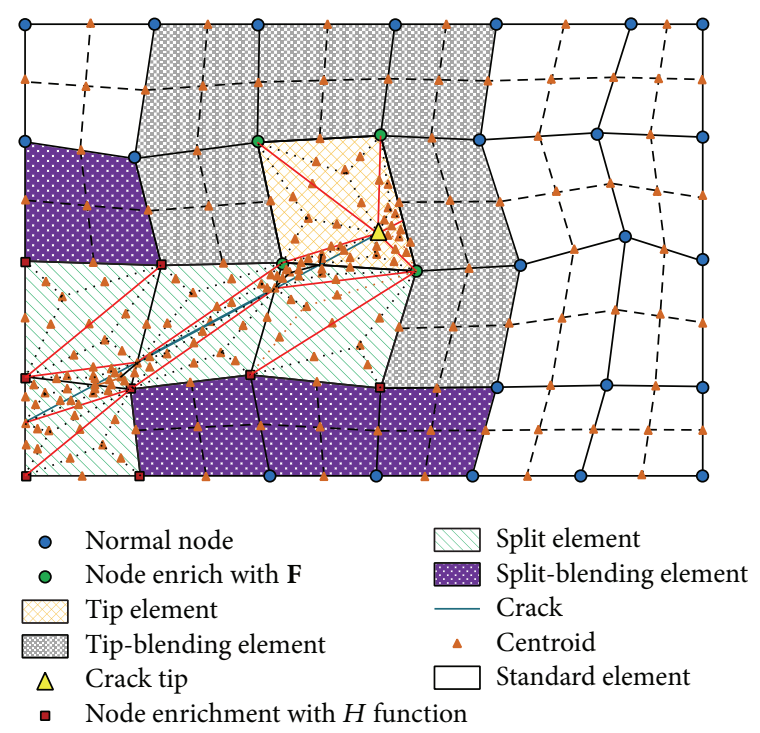

FIGURE 2: Classification of smoothing domains in CS-XFEM.

and circles, respectively. The support domain of $N^{\text {CS-FEM }}$ is associated with nodes of CS-FEM, shown in Figure 2.

As illustrated in Figure 2, four-node quadrilateral cells are used for cell-based strain smoothing operation. The meshing characteristics of CS-XFEM are consistent with XFEM. The complex structure can adopt the fine mesh. For CS-FEM, the number of subcells (SC) would affect the performance of the results. In the case that the solution of SFEM (SC $=1$ ) is overestimated to the exact solution, there exists one optimal value $\mathrm{SC}>1$ (normally $\mathrm{SC}=4$ ) which gives the best results as compared to the exact ones. In the case that the solution of SFEM $(\mathrm{SC}=1)$ is underestimated to the exact solution, it is suggested that we should use $\mathrm{SC}=2$ to obtain the solution. This solution will be stable and have the smallest displacement and energy norms. In practical calculation, we can use $\mathrm{SC}=4$ for all problems. The results will always be better than that of standard FEM. And in many cases (not all) this solution $(\mathrm{SC}=1)$ is closest to the exact solution [21]. So we can use four subcells for each quadrilateral.

In CS-XFEM, Heaviside enriched degrees of freedom are added to nodes in $N^{\text {CS-c }}$ whose support domain is split by the crack and tip enriched degrees of freedom are added to nodes in set $N^{\mathrm{CS}-f}$ whose support domain contains the crack tip. In order to keep the convergence rate as high as possible, a socalled geometric enrichment which is independent from the discretization is used [45-47]:

$$
H(\mathbf{x})= \begin{cases}1 & \left(\mathbf{x}-\mathbf{x}^{*}\right) \cdot \mathbf{n} \geq 0 \\ -1 & \text { otherwise }\end{cases}
$$

where $\mathbf{x}^{*}$ is a point on the crack surface; see Figure 3.

For piezoelectric problems, it is advisable to use the regular enrichment functions stemming from the isotropic elasticity. It should be mentioned that similar results have been obtained independently with alternative enrichment functions for cracks in confined plasticity problems [42]. The 


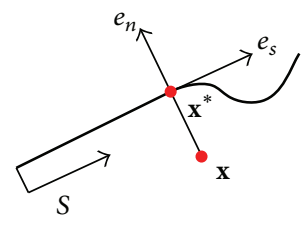

FIGURE 3: Normal and tangential coordinates for a crack.

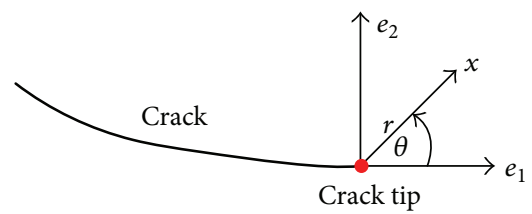

Figure 4: Polar coordinate system associated with a crack tip.

near-tip enrichment consists of functions which incorporate the radial and angular behaviors of the two-dimensional asymptotic crack tip displacement field $[37,48]$ :

$$
\begin{aligned}
& \left\{\mathbf{F}_{l}\right\}_{1 \leq l \leq 4} \\
& =\sqrt{r}\left\{\sin \left(\frac{\theta}{2}\right) \cos \left(\frac{\theta}{2}\right) \cos (\theta) \sin \left(\frac{\theta}{2}\right) \sin (\theta) \cos \left(\frac{\theta}{2}\right)\right\},
\end{aligned}
$$

where $r$ and $\theta$ are polar coordinates in the local crack tip coordinate system; see Figure 4.

Employing the strain smoothing operation, the smoothed strain in the domain $\Omega_{c}$ from the displacement approximation in (8) can be written in the following matrix form:

$$
\begin{aligned}
\overline{\boldsymbol{\varepsilon}}_{k}= & \sum_{I \in N^{\mathrm{CS}-\mathrm{FEM}}} \overline{\mathbf{B}}_{I}^{u}\left(\mathbf{x}_{k}\right) \mathbf{u}_{I} \\
& +\sum_{J \in N^{\mathrm{CS}-\mathrm{c}}} \overline{\mathbf{B}}_{J}^{a}\left(\mathbf{x}_{k}\right)\left(H(\mathbf{x})-H\left(\mathbf{x}_{J}\right)\right) \mathbf{a}_{J} \\
& +\sum_{K \in N^{\mathrm{CS}-f}} \overline{\mathbf{B}}_{K}^{b}\left(\mathbf{x}_{k}\right) \sum_{l=1}^{4}\left(\mathbf{F}_{l}(\mathbf{x})-\mathbf{F}_{l}\left(\mathbf{x}_{K}\right)\right) \mathbf{b}_{K}^{l},
\end{aligned}
$$

where $\overline{\mathbf{B}}_{I}^{u}\left(\mathbf{x}_{k}\right)$ is the smoothed strain gradient matrix for the standard CS-FEM part; $\overline{\mathbf{B}}_{J}^{a}\left(\mathbf{x}_{k}\right)$ and $\overline{\mathbf{B}}_{K}^{b}\left(\mathbf{x}_{k}\right)$ correspond to the enriched parts of the smoothed strain gradient matrix associated with the Heaviside and branch functions, respectively.

When employing the electric field smoothing operation, the smoothed electric field in the domain $\Omega_{c}$ from the displacement approximation in (9) can be written in the following matrix form:

$$
\begin{aligned}
\overline{\mathbf{E}}_{k}= & \sum_{I \in N^{\text {CS-FFM }}} \overline{\mathbf{B}}_{I}^{\phi}\left(\mathbf{x}_{k}\right) \mathbf{u}_{I} \\
& +\sum_{J \in N^{\text {CS-c }}} \overline{\mathbf{B}}_{J}^{\alpha}\left(\mathbf{x}_{k}\right)\left(H(\mathbf{x})-H\left(\mathbf{x}_{J}\right)\right) \boldsymbol{\alpha}_{J} \\
& +\sum_{K \in N^{\text {CS }-f}} \overline{\mathbf{B}}_{K}^{\beta}\left(\mathbf{x}_{k}\right) \sum_{l=1}^{4}\left(\mathbf{F}_{l}(\mathbf{x})-\mathbf{F}_{l}\left(\mathbf{x}_{K}\right)\right) \boldsymbol{\beta}_{K}^{l},
\end{aligned}
$$

where $\overline{\mathbf{B}}_{I}^{\phi}\left(\mathbf{x}_{k}\right)$ is the smoothed electric field gradient matrix for the standard CS-FEM part; $\overline{\mathbf{B}}_{J}^{\alpha}\left(\mathbf{x}_{k}\right)$ and $\overline{\mathbf{B}}_{K}^{\beta}\left(\mathbf{x}_{k}\right)$ correspond to the enriched parts of the smoothed electric field gradient matrix associated with the Heaviside and branch functions, respectively. These matrixes can be written as follows:

$$
\begin{aligned}
& \overline{\mathbf{B}}_{I}^{r}\left(\mathbf{x}_{k}\right)=\left[\begin{array}{cc}
b_{I x}^{r}\left(\mathbf{x}_{k}\right) & 0 \\
0 & b_{I y}^{r}\left(\mathbf{x}_{k}\right) \\
b_{I y}^{r}\left(\mathbf{x}_{k}\right) & b_{I x}^{r}\left(\mathbf{x}_{k}\right)
\end{array}\right], \quad r=u, a, b, \\
& \overline{\mathbf{B}}_{I}^{t}\left(\mathbf{x}_{k}\right)=\left[\begin{array}{c}
b_{I x}^{t}\left(\mathbf{x}_{k}\right) \\
b_{I y}^{t}\left(\mathbf{x}_{k}\right)
\end{array}\right], \quad t=\phi, \alpha, \phi,
\end{aligned}
$$

where

$$
\begin{aligned}
& b_{I h}^{u}\left(\mathbf{x}_{k}\right)=\frac{1}{A_{k}^{s}} \int_{\Gamma_{k}^{s}} n_{h}\left(\mathbf{x}_{k}\right) N_{I}^{u} d \Gamma^{s}=\sum_{m=1}^{N_{\text {seg }}}\left(\sum_{n=1}^{N_{\text {gau }}} n_{h}\left(\mathbf{x}_{m, n}\right)\right. \\
& \left.\cdot N_{I}^{u}\left(\mathbf{x}_{m, n}\right) w_{m, n}\right), \quad h=x, z \\
& b_{I h}^{a}\left(\mathbf{x}_{k}\right)=\frac{1}{A_{k}^{s}} \int_{\Gamma_{k}^{s}} n_{h}\left(\mathbf{x}_{k}\right) N_{I}^{u}\left(H(\mathbf{x})-H\left(\mathbf{x}_{I}\right)\right) d \Gamma^{s} \\
& =\sum_{m=1}^{N_{\text {seg }}}\left(\sum_{n=1}^{N_{\text {gau }}} n_{h}\left(\mathbf{x}_{m, n}\right) N_{I}^{u}\left(\mathbf{x}_{m, n}\right)\left(H\left(\mathbf{x}_{m, n}\right)-H\left(\mathbf{x}_{I}\right)\right)\right. \\
& \left.\cdot w_{m, n}\right), \quad h=x, z \\
& b_{I h}^{b}\left(\mathbf{x}_{k}\right)=\frac{1}{A_{k}^{s}} \int_{\Gamma_{k}^{s}} n_{h}\left(\mathbf{x}_{k}\right) N_{I}^{u} \sum_{l=1}^{4}\left(\mathbf{F}_{l}(\mathbf{x})-\mathbf{F}_{l}\left(\mathbf{x}_{K}\right)\right) d \Gamma^{s} \\
& =\sum_{m=1}^{N_{\text {seg }}}\left(\sum_{n=1}^{N_{\text {gau }}} n_{h}\left(\mathbf{x}_{m, n}\right) N_{I}^{u}\left(\mathbf{x}_{m, n}\right)\left(\mathbf{F}_{l}\left(\mathbf{x}_{m, n}\right)-\mathbf{F}_{l}\left(\mathbf{x}_{I}\right)\right)\right. \\
& \left.\cdot w_{m, n}\right), \quad h=x, z, l=1,2,3,4 \\
& b_{I h}^{\phi}\left(\mathbf{x}_{k}\right)=\frac{1}{A_{k}^{s}} \int_{\Gamma_{k}^{s}} n_{h}\left(\mathbf{x}_{k}\right) N_{I} d \Gamma^{s}=\sum_{m=1}^{N_{\text {seg }}}\left(\sum_{n=1}^{N_{\text {gau }}} n_{h}\left(\mathbf{x}_{m, n}\right)\right. \\
& \left.\cdot N_{I}^{\phi}\left(\mathbf{x}_{m, n}\right) w_{m, n}\right), \quad h=x, z \\
& b_{I h}^{\alpha}\left(\mathbf{x}_{k}\right)=\frac{1}{A_{k}^{s}} \int_{\Gamma_{k}^{s}} n_{h}\left(\mathbf{x}_{k}\right) N_{I}^{\phi}\left(H(\mathbf{x})-H\left(\mathbf{x}_{I}\right)\right) d \Gamma^{s} \\
& =\sum_{m=1}^{N_{\text {seg }}}\left(\sum_{n=1}^{N_{\text {gau }}} n_{h}\left(\mathbf{x}_{m, n}\right) N_{I}^{\phi}\left(\mathbf{x}_{m, n}\right)\left(H\left(\mathbf{x}_{m, n}\right)-H\left(\mathbf{x}_{I}\right)\right)\right. \\
& \left.\cdot w_{m, n}\right), \quad h=x, z
\end{aligned}
$$




$$
\begin{aligned}
& b_{I h}^{\beta}\left(\mathbf{x}_{k}\right)=\frac{1}{A_{k}^{s}} \int_{\Gamma_{k}^{s}} n_{h}\left(\mathbf{x}_{k}\right) N_{I}^{\phi} \sum_{l=1}^{4}\left(\mathbf{F}_{l}(\mathbf{x})-\mathbf{F}_{l}\left(\mathbf{x}_{K}\right)\right) d \Gamma^{s} \\
& =\sum_{m=1}^{N_{\text {seg }}}\left(\sum_{n=1}^{N_{\text {gau }}} n_{h}\left(\mathbf{x}_{m, n}\right) N_{I}^{\phi}\left(\mathbf{x}_{m, n}\right)\left(\mathbf{F}_{l}\left(\mathbf{x}_{m, n}\right)-\mathbf{F}_{l}\left(\mathbf{x}_{I}\right)\right)\right. \\
& \left.\cdot w_{m, n}\right), \quad h=x, z, l=1,2,3,4,
\end{aligned}
$$

where $N_{\text {seg }}$ is the number of segments of the boundary $\Gamma_{k}^{s}$, $N_{\text {gau }}$ is the number of Gauss points used in each segment, $w_{m, n}$ is the corresponding Gauss weights, $n_{x}$ and $n_{z}$ are the outward unit normal components to each segment on the smoothing domain boundary, and $x_{m, n}$ is the $n$th Gaussian point on the $m$ th segment of the boundary $\Gamma_{k}^{s}$.

The standard discrete system of equations is obtained:

$$
\begin{aligned}
& {\left[\begin{array}{lll}
\mathbf{K}_{I J}^{u u} & \mathbf{K}_{I J}^{u a} & \mathbf{K}_{I J}^{u b} \\
\mathbf{K}_{I J}^{a u} & \mathbf{K}_{I J}^{a a} & \mathbf{K}_{I J}^{a b} \\
\mathbf{K}_{I J}^{b u} & \mathbf{K}_{I J}^{b a} & \mathbf{K}_{I J}^{b b}
\end{array}\right]\left\{\begin{array}{l}
\mathbf{u} \\
\mathbf{a} \\
\mathbf{b}
\end{array}\right\}+\left[\begin{array}{lll}
\mathbf{K}_{I J}^{u \phi} & \mathbf{K}_{I J}^{u \alpha} & \mathbf{K}_{I J}^{u \beta} \\
\mathbf{K}_{I J}^{a \phi} & \mathbf{K}_{I J}^{\alpha \alpha} & \mathbf{K}_{I J}^{\alpha \beta} \\
\mathbf{K}_{I J}^{b \phi} & \mathbf{K}_{I J}^{b \alpha} & \mathbf{K}_{I J}^{b \beta}
\end{array}\right]\left\{\begin{array}{l}
\boldsymbol{\Phi} \\
\boldsymbol{\alpha} \\
\boldsymbol{\beta}
\end{array}\right\}} \\
& =\left\{\begin{array}{c}
\mathbf{f} \\
\mathbf{f}^{a} \\
\mathbf{f}^{b}
\end{array}\right\}, \\
& {\left[\begin{array}{lll}
\mathbf{K}_{I J}^{\phi u} & \mathbf{K}_{I J}^{\phi a} & \mathbf{K}_{I J}^{\phi b} \\
\mathbf{K}_{I J}^{\alpha u} & \mathbf{K}_{I J}^{\alpha a} & \mathbf{K}_{I J}^{\alpha b} \\
\mathbf{K}_{I J}^{\beta u} & \mathbf{K}_{I J}^{\beta a} & \mathbf{K}_{I J}^{\beta b}
\end{array}\right]\left\{\begin{array}{l}
\mathbf{u} \\
\mathbf{a} \\
\mathbf{b}
\end{array}\right\}-\left[\begin{array}{ccc}
\mathbf{K}_{I J}^{\phi \phi} & \mathbf{K}_{I J}^{\phi \alpha} & \mathbf{K}_{I I}^{\phi \beta} \\
\mathbf{K}_{I J}^{\alpha \phi} & \mathbf{K}_{I J}^{\alpha \alpha} & \mathbf{K}_{I J}^{\alpha \beta} \\
\mathbf{K}_{I J}^{\beta \phi} & \mathbf{K}_{I J}^{\beta \alpha} & \mathbf{K}_{I J}^{\beta \beta}
\end{array}\right]\left\{\begin{array}{c}
\boldsymbol{\Phi} \\
\boldsymbol{\alpha} \\
\boldsymbol{\beta}
\end{array}\right\}} \\
& =\left\{\begin{array}{c}
\mathbf{g} \\
\mathbf{g}^{a} \\
\mathbf{g}^{b}
\end{array}\right\}
\end{aligned}
$$

where

$$
\begin{aligned}
\mathbf{K}_{I J}^{u u} & =\sum_{k=1}^{N_{s}} \int_{\Omega_{k}^{s}}\left(\overline{\mathbf{B}}_{I}^{u}\right)^{T} \mathbf{C} \overline{\mathbf{B}}_{J}^{u} d \Omega=\sum_{k=1}^{N_{s}}\left(\overline{\mathbf{B}}_{I}^{u}\right)^{T} \mathbf{C} \overline{\mathbf{B}}_{J}^{u} A_{k}^{s}, \\
\mathbf{K}_{I J}^{u a} & =\sum_{k=1}^{N_{s}} \int_{\Omega_{k}^{s}}\left(\overline{\mathbf{B}}_{I}^{u}\right)^{T} \mathbf{C} \overline{\mathbf{B}}_{J}^{a} d \Omega=\sum_{k=1}^{N_{s}}\left(\overline{\mathbf{B}}_{I}^{u}\right)^{T} \mathbf{C} \overline{\mathbf{B}}_{J}^{a} A_{k}^{s} \\
& =\left(\mathbf{K}_{I J}^{a u}\right)^{T}, \\
\mathbf{K}_{I J}^{u b} & =\sum_{k=1}^{N_{s}} \int_{\Omega_{k}^{s}}\left(\overline{\mathbf{B}}_{I}^{u}\right)^{T} \mathbf{C} \overline{\mathbf{B}}_{J}^{b} d \Omega=\sum_{k=1}^{N_{s}}\left(\overline{\mathbf{B}}_{I}^{u}\right)^{T} \mathbf{C} \overline{\mathbf{B}}_{J}^{b} A_{k}^{s} \\
& =\left(\mathbf{K}_{I J}^{b u}\right)^{T}, \\
\mathbf{K}_{I J}^{a a} & =\sum_{k=1}^{N_{s}} \int_{\Omega_{k}^{s}}\left(\overline{\mathbf{B}}_{I}^{a}\right)^{T} \mathbf{C} \overline{\mathbf{B}}_{J}^{a} d \Omega=\sum_{k=1}^{N_{s}}\left(\overline{\mathbf{B}}_{I}^{a}\right)^{T} \mathbf{C} \overline{\mathbf{B}}_{J}^{a} A_{k}^{s},
\end{aligned}
$$

$$
\begin{aligned}
\mathbf{K}_{I J}^{a b} & =\sum_{k=1}^{N_{s}} \int_{\Omega_{k}^{s}}\left(\overline{\mathbf{B}}_{I}^{a}\right)^{T} \mathbf{C} \overline{\mathbf{B}}_{J}^{b} d \Omega=\sum_{k=1}^{N_{s}}\left(\overline{\mathbf{B}}_{I}^{a}\right)^{T} \mathbf{C} \overline{\mathbf{B}}_{J}^{b} A_{k}^{s} \\
& =\left(\mathbf{K}_{I J}^{b a}\right)^{T}, \\
\mathbf{K}_{I J}^{b b} & =\sum_{k=1}^{N_{s}} \int_{\Omega_{k}^{s}}\left(\overline{\mathbf{B}}_{I}^{b}\right)^{T} \mathbf{C} \overline{\mathbf{B}}_{J}^{b} d \Omega=\sum_{k=1}^{N_{s}}\left(\overline{\mathbf{B}}_{I}^{b}\right)^{T} \mathbf{C} \overline{\mathbf{B}}_{J}^{b} A_{k}^{s}, \\
\mathbf{K}_{I J}^{u \phi} & =\sum_{k=1}^{N_{s}} \int_{\Omega_{k}^{s}}\left(\overline{\mathbf{B}}_{I}^{u}\right)^{T} \mathbf{e} \overline{\mathbf{B}}_{J}^{\phi} d \Omega=\sum_{k=1}^{N_{s}}\left(\overline{\mathbf{B}}_{I}^{u}\right)^{T} \mathbf{e} \overline{\mathbf{B}}_{J}^{\phi} A_{k}^{s} \\
& =\left(\mathbf{K}_{I J}^{\phi u}\right)^{T}, \\
\mathbf{K}_{I J}^{u \alpha} & =\sum_{k=1}^{N_{s}} \int_{\Omega_{k}^{s}}\left(\overline{\mathbf{B}}_{I}^{u}\right)^{T} \mathbf{e} \overline{\mathbf{B}}_{J}^{\alpha} d \Omega=\sum_{k=1}^{N_{s}}\left(\overline{\mathbf{B}}_{I}^{u}\right)^{T} \mathbf{e} \overline{\mathbf{B}}_{J}^{\alpha} A_{k}^{s} \\
& =\left(\mathbf{K}_{I J}^{\alpha u}\right)^{T},
\end{aligned}
$$$$
\mathbf{K}_{I J}^{u \beta}=\sum_{k=1}^{N_{s}} \int_{\Omega_{k}^{s}}\left(\overline{\mathbf{B}}_{I}^{u}\right)^{T} \mathbf{e} \overline{\mathbf{B}}_{J}^{\beta} d \Omega=\sum_{k=1}^{N_{s}}\left(\overline{\mathbf{B}}_{I}^{u}\right)^{T} \mathbf{e} \overline{\mathbf{B}}_{J}^{\beta} A_{k}^{s}
$$$$
=\left(\mathbf{K}_{I J}^{\beta u}\right)^{T} \text {, }
$$$$
\mathbf{K}_{I J}^{a \phi}=\sum_{k=1}^{N_{s}} \int_{\Omega_{k}^{s}}\left(\overline{\mathbf{B}}_{I}^{a}\right)^{T} \mathbf{e} \overline{\mathbf{B}}_{J}^{\phi} d \Omega=\sum_{k=1}^{N_{s}}\left(\overline{\mathbf{B}}_{I}^{a}\right)^{T} \mathbf{e} \overline{\mathbf{B}}_{J}^{\phi} A_{k}^{s}
$$$$
=\left(\mathbf{K}_{I J}^{\phi a}\right)^{T} \text {, }
$$$$
\mathbf{K}_{I J}^{\alpha \alpha}=\sum_{k=1}^{N_{s}} \int_{\Omega_{k}^{s}}\left(\overline{\mathbf{B}}_{I}^{a}\right)^{T} \mathbf{e} \overline{\mathbf{B}}_{J}^{\alpha} d \Omega=\sum_{k=1}^{N_{s}}\left(\overline{\mathbf{B}}_{I}^{a}\right)^{T} \mathbf{e} \overline{\mathbf{B}}_{J}^{\alpha} A_{k}^{s}
$$$$
=\left(\mathbf{K}_{I J}^{u a}\right)^{T} \text {, }
$$$$
\mathbf{K}_{I J}^{a \beta}=\sum_{k=1}^{N_{s}} \int_{\Omega_{k}^{s}}\left(\overline{\mathbf{B}}_{I}^{a}\right)^{T} \mathbf{e} \overline{\mathbf{B}}_{J}^{\beta} d \Omega=\sum_{k=1}^{N_{s}}\left(\overline{\mathbf{B}}_{I}^{a}\right)^{T} \mathbf{e} \overline{\mathbf{B}}_{J}^{\beta} A_{k}^{s}
$$$$
=\left(\mathbf{K}_{I J}^{\beta a}\right)^{T} \text {, }
$$$$
\mathbf{K}_{I J}^{b \phi}=\sum_{k=1}^{N_{s}} \int_{\Omega_{k}^{s}}\left(\overline{\mathbf{B}}_{I}^{b}\right)^{T} \mathbf{e} \overline{\mathbf{B}}_{J}^{\phi} d \Omega=\sum_{k=1}^{N_{s}}\left(\overline{\mathbf{B}}_{I}^{b}\right)^{T} \mathbf{e} \overline{\mathbf{B}}_{J}^{\phi} A_{k}^{s}
$$$$
=\left(\mathbf{K}_{I J}^{\phi b}\right)^{T} \text {, }
$$$$
\mathbf{K}_{I J}^{b \alpha}=\sum_{k=1}^{N_{s}} \int_{\Omega_{k}^{s}}\left(\overline{\mathbf{B}}_{I}^{b}\right)^{T} \mathbf{e} \overline{\mathbf{B}}_{J}^{\alpha} d \Omega=\sum_{k=1}^{N_{s}}\left(\overline{\mathbf{B}}_{I}^{b}\right)^{T} \mathbf{e} \overline{\mathbf{B}}_{J}^{\alpha} A_{k}^{s}
$$$$
=\left(\mathbf{K}_{I J}^{u b}\right)^{T} \text {, }
$$$$
\mathbf{K}_{I J}^{b \beta}=\sum_{k=1}^{N_{s}} \int_{\Omega_{k}^{s}}\left(\overline{\mathbf{B}}_{I}^{b}\right)^{T} \mathbf{e} \overline{\mathbf{B}}_{J}^{\beta} d \Omega=\sum_{k=1}^{N_{s}}\left(\overline{\mathbf{B}}_{I}^{b}\right)^{T} \mathbf{e} \overline{\mathbf{B}}_{J}^{\beta} A_{k}^{s}
$$$$
=\left(\mathbf{K}_{I J}^{\beta b}\right)^{T} \text {, }
$$ 


$$
\begin{aligned}
\mathbf{K}_{I J}^{\phi \phi} & =\sum_{k=1}^{N_{s}} \int_{\Omega_{k}^{s}}\left(\overline{\mathbf{B}}_{I}^{\phi}\right)^{T} \boldsymbol{\kappa} \overline{\mathbf{B}}_{J}^{\phi} d \Omega=\sum_{k=1}^{N_{s}}\left(\overline{\mathbf{B}}_{I}^{\phi}\right)^{T} \boldsymbol{\kappa} \overline{\mathbf{B}}_{J}^{\phi} A_{k}^{s}, \\
\mathbf{K}_{I J}^{\phi \alpha} & =\sum_{k=1}^{N_{s}} \int_{\Omega_{k}^{s}}\left(\overline{\mathbf{B}}_{I}^{\phi}\right)^{T} \boldsymbol{\kappa} \overline{\mathbf{B}}_{J}^{\alpha} d \Omega=\sum_{k=1}^{N_{s}}\left(\overline{\mathbf{B}}_{I}^{\phi}\right)^{T} \boldsymbol{\kappa} \overline{\mathbf{B}}_{J}^{\alpha} A_{k}^{s} \\
& =\left(\mathbf{K}_{I J}^{\alpha \phi}\right)^{T}, \\
\mathbf{K}_{I J}^{\phi \beta} & =\sum_{k=1}^{N_{s}} \int_{\Omega_{k}^{s}}\left(\overline{\mathbf{B}}_{I}^{\phi}\right)^{T} \boldsymbol{\kappa} \overline{\mathbf{B}}_{J}^{\beta} d \Omega=\sum_{k=1}^{N_{s}}\left(\overline{\mathbf{B}}_{I}^{\phi}\right)^{T} \boldsymbol{\kappa} \overline{\mathbf{B}}_{J}^{\beta} A_{k}^{s} \\
& =\left(\mathbf{K}_{I J}^{\beta \phi}\right)^{T}, \\
\mathbf{K}_{I J}^{\alpha \alpha} & =\sum_{k=1}^{N_{s}} \int_{\Omega_{k}^{s}}\left(\overline{\mathbf{B}}_{I}^{\alpha}\right)^{T} \boldsymbol{\kappa} \overline{\mathbf{B}}_{J}^{\alpha} d \Omega=\sum_{k=1}^{N_{s}}\left(\overline{\mathbf{B}}_{I}^{\alpha}\right)^{T} \boldsymbol{\kappa} \overline{\mathbf{B}}_{J}^{\alpha} A_{k}^{s}, \\
\mathbf{K}_{I J}^{\alpha \beta} & =\sum_{k=1}^{N_{s}} \int_{\Omega_{k}^{s}}\left(\overline{\mathbf{B}}_{I}^{\alpha}\right)^{T} \boldsymbol{\kappa} \overline{\mathbf{B}}_{J}^{\beta} d \Omega=\sum_{k=1}^{N_{s}}\left(\overline{\mathbf{B}}_{I}^{\alpha}\right)^{T} \boldsymbol{\kappa} \overline{\mathbf{B}}_{J}^{\beta} A_{k}^{s} \\
& =\left(\mathbf{K}_{I J}^{\beta \alpha}\right)^{T}, \\
\mathbf{K}_{I J}^{\beta \beta} & =\sum_{k=1}^{N_{s}} \int_{\Omega_{k}^{s}}\left(\overline{\mathbf{B}}_{I}^{\beta}\right)^{T} \boldsymbol{\kappa} \overline{\mathbf{B}}_{J}^{\beta} d \Omega=\sum_{k=1}^{N_{s}}\left(\overline{\mathbf{B}}_{I}^{\beta}\right)^{T} \boldsymbol{\kappa} \overline{\mathbf{B}}_{J}^{\beta} A_{k}^{s} .
\end{aligned}
$$

\section{Electromechanical $J$-Integral}

According to research by Rice [49] and Eshelby [50] on pure mechanical applications,

$$
\begin{aligned}
\mathbf{F}_{k} & =\oint_{s}\left(H \delta_{k j}-\sigma_{i j} u_{i, k}+D_{j} E_{k}\right) n_{j} d s \\
H & =\frac{1}{2}\left(\sigma_{i j} \varepsilon_{i j}-D_{i} E_{i}\right)
\end{aligned}
$$

Equation (18) describes the material force when an electromechanically loaded domain is virtually displaced by the vector $\delta_{x j}$. The term in brackets is called the piezoelectric energy momentum tensor:

$$
Q_{k j}=H \delta_{k j}-\sigma_{i j} u_{i, k}+D_{j} E_{k}
$$

analogous to the energy momentum tensor, which was introduced by Eshelby [50]. If the integration path $S$ contains neither defects nor source terms $b_{i}$ and $\omega_{\Omega}$, the force vanishes and $\mathbf{F}_{k} \equiv 0$. Now we consider a path $\Gamma_{\varepsilon}$ enclosing the tip of a crack; see Figure 5. The electromechanical $J_{k}^{\mathrm{em}}$-integral vector is the material force associated with the crack tip singularity. It is defined as the limit when $\Gamma_{\varepsilon}$ is shrunk towards $r \rightarrow 0$

$$
\begin{aligned}
J_{k}^{\mathrm{em}} & =\lim _{r \rightarrow 0} \int_{\Gamma_{\varepsilon}} Q_{k j} n_{j} d s \\
& =\lim _{r \rightarrow 0} \int_{\Gamma_{\varepsilon}}\left(H \delta_{k j}-\sigma_{i j} u_{i, k}+D_{j} E_{k}\right) n_{j} d s .
\end{aligned}
$$

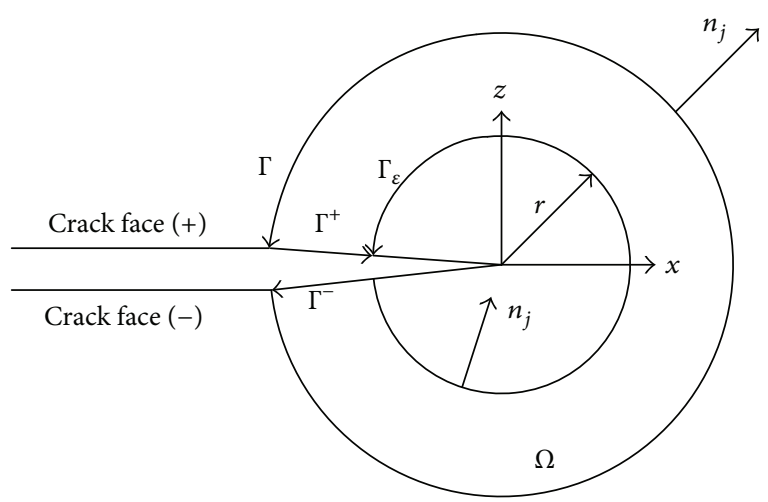

FIGURE 5: Combined integration path around a crack tip.

For the numerical analysis of the $J_{k}^{\mathrm{em}}$-integral, an equivalent domain integral is easier to handle than a line integral. The condition for the transformation into a domain integral applying the Gaussian integral theorem, a closed integration path $S=\Gamma-\Gamma_{\varepsilon}+\Gamma^{+}+\Gamma^{-}$with an outward pointing normal vector is given, as can be seen in Figure 5. Then (21) can be written as follows:

$$
J_{k}^{\mathrm{em}}=-\int_{s} Q_{k j} n_{j} d s+\int_{\Gamma+\Gamma^{+}+\Gamma^{-}} Q_{k j} n_{j} d s .
$$

Now a weighting function $q$ is introduced, which should be continuous and satisfy the condition

$$
q= \begin{cases}0 & \text { on } \Gamma \\ 1 & \text { on } \Gamma_{\varepsilon} .\end{cases}
$$

The calculation of the $J$-integral is carried out in a ring of elements surrounding the crack tip. The elements within the ring move as a rigid body. $q$ is a constant in these elements; so the derivative of $q$ with respect to $x_{j}$ is zero. For the elements outside the ring, $q$ is zero, and again the derivative of $q$ is zero. For the elements belonging to the ring, the vector $q$ is 1 . If the weighting function $q$ is introduced in (22), the contribution of the integral along $\Gamma$ disappears and

$$
J_{k}^{\mathrm{em}}=-\int_{s} Q_{k j} n_{j} q d s+\int_{\Gamma^{+}+\Gamma^{-}} Q_{k j} n_{j} q d s .
$$

The transformation into an equivalent domain integral leads to

$$
J_{k}^{\mathrm{em}}=-\int_{\Omega}\left(Q_{k j} q\right)_{, j} d \Omega+\int_{\Gamma^{+}+\Gamma^{-}} Q_{k j} n_{j} q d s .
$$

If we use the expression of $Q_{k j}$ for (20), we get the 2dimensional $J_{k}^{\mathrm{em}}$-integral as a domain integral:

$$
\begin{aligned}
J_{k}^{\mathrm{em}}= & -\int_{\Omega}\left(H \delta_{k j}-\sigma_{i j} u_{i, k}+D_{j} E_{k}\right) q_{, j} d \Omega \\
& -\int_{\Omega}\left(\left.H_{, k}\right|_{\exp }-b_{i} u_{i, k}+\omega_{\Omega} E_{k}\right) q d \Omega \\
& +\int_{\Gamma^{+}+\Gamma^{-}}\left(H n_{k}-T_{i} u_{i, k}-\omega_{s} E_{k}\right) q d s .
\end{aligned}
$$


If the material is homogenous, no volume forces or charges and no crack face tractions or charges are applied, and the crack face normal points to the $z$-directions; that is,

$$
\begin{aligned}
\left.H_{, k}\right|_{\text {exp }} & =0, \\
b_{i} & =0, \\
\omega_{\Omega} & =0, \\
T_{i} & =0, \\
\omega_{s} & =0, \\
n_{k} & =(0, \pm 1)^{T} .
\end{aligned}
$$

Equation (26) can be reduced as

$$
\begin{aligned}
J_{k}^{\mathrm{em}}= & -\int_{\Omega}\left(H \delta_{k j}-\sigma_{i j} u_{i, k}+D_{j} E_{k}\right) q_{, j} d \Omega \\
& +\int_{\Gamma^{+}+\Gamma^{-}}\left(H n_{k}\right) q d s .
\end{aligned}
$$

The $x$-component of the electromechanical $J_{k}^{\mathrm{em}}$-integral vector has a physical meaning of the energy release rate $G=J_{1}^{\mathrm{em}}$.

The mechanical stresses and the electric fluxes behave singular as $1 / \sqrt{r}$, whereas the electric potential and the mechanical displacements show a parabolic shape $\sim \sqrt{r}$. The angular functions $f_{i j}, g_{i j}, d_{j}$, and $v_{j}$ depend only on material constants. The coefficients $K_{\mathrm{I}}, K_{\mathrm{II}}$, and $K_{\mathrm{III}}$ are the well-known mechanical stress intensity factors, which are complemented by the new forth "electric intensity factor" $K_{\mathrm{D}}$, that characterizes the electric field singularity. Mutual interdependence between mechanical and electrical crack tip parameters can be given by

$$
\begin{aligned}
& \sigma_{i j}(r, \theta)=\frac{1}{\sqrt{r}}\left[K_{\mathrm{I}} f_{i j}^{\mathrm{I}}(\theta)+K_{\mathrm{II}} f_{i j}^{\mathrm{II}}(\theta)+K_{\mathrm{III}} f_{i j}^{\mathrm{III}}(\theta)\right. \\
& \left.\quad+K_{\mathrm{D}} f_{i j}^{\mathrm{D}}(\theta)\right], \\
& u_{i j}(r, \theta)=\frac{1}{\sqrt{r}}\left[K_{\mathrm{I}} g_{i j}^{\mathrm{I}}(\theta)+K_{\mathrm{II}} g_{i j}^{\mathrm{II}}(\theta)+K_{\mathrm{III}} g_{i j}^{\mathrm{III}}(\theta)\right. \\
& \left.\quad+K_{\mathrm{D}} g_{i j}^{\mathrm{D}}(\theta)\right], \\
& D_{j}(r, \theta)=\frac{1}{\sqrt{r}}\left[K_{\mathrm{I}} d_{j}^{\mathrm{I}}(\theta)+K_{\mathrm{II}} d_{j}^{\mathrm{II}}(\theta)+K_{\mathrm{III}} d_{j}^{\mathrm{III}}(\theta)\right. \\
& \left.\quad+K_{\mathrm{D}} d_{j}^{\mathrm{D}}(\theta)\right], \\
& \varphi(r, \theta)=\frac{1}{\sqrt{r}}\left[K_{\mathrm{I}} v_{j}^{\mathrm{I}}(\theta)+K_{\mathrm{II}} v_{j}^{\mathrm{II}}(\theta)+K_{\mathrm{III}} v_{j}^{\mathrm{III}}(\theta)\right. \\
& \left.\quad+K_{\mathrm{D}} v_{j}^{\mathrm{D}}(\theta)\right] .
\end{aligned}
$$

If the limit to an infinitesimal crack growth is considered taking place inside the near-tip solution, its relationship with the intensity factors can be found in [6]. Consider

$$
\begin{aligned}
G & =\frac{1}{2}\{\mathbf{K}\}^{T}[\mathbf{Y}(C, e, \kappa)]\{\mathbf{K}\}, \\
\{\mathbf{K}\} & =\left\{\begin{array}{lll}
\left.K_{\mathrm{II}} K_{\mathrm{I}} K_{\mathrm{III}} K_{\mathrm{D}}\right\} .
\end{array}\right.
\end{aligned}
$$

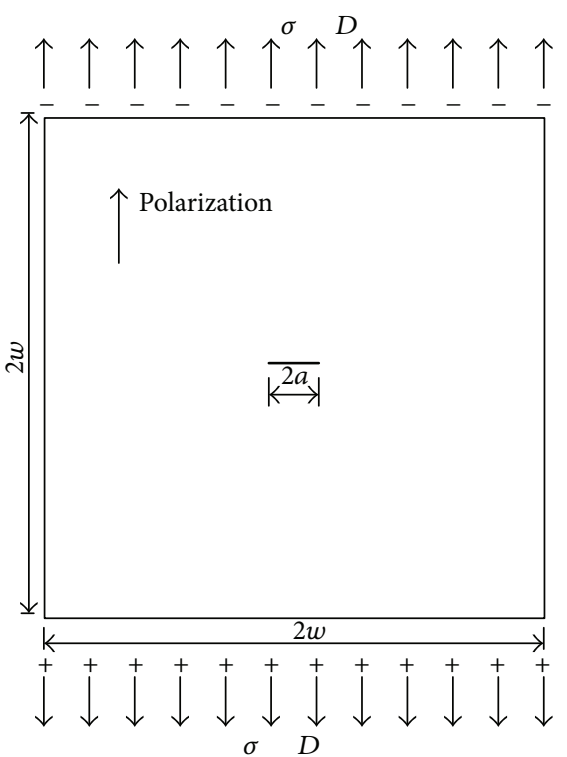

FIgURE 6: Electromechanical Griffith crack (uniaxial load).

The generalized Irwin-matrix $[\mathbf{Y}]$ depends on the elastic, piezoelectric, and dielectric material constants and the relative orientation of the crack with respect to material's axes and polarization vectors. For a special case when the crack lies perpendicular to the polarization, the crack tip field and the coefficients of $[\mathbf{Y}]$ were determined in $[10,11]$. Then $G$ is reduced to the following expression, where, $C_{L}, C_{T}, C_{A}, e$, and $\kappa$ are material constants:

$$
\begin{aligned}
G & =G_{m}^{\mathrm{I}}+G_{m}^{\mathrm{II}}+G_{m}^{\mathrm{III}}+G_{\mathrm{D}} \\
& =\frac{1}{2}\left\{\frac{K_{\mathrm{I}}^{2}}{C_{T}}+\frac{K_{\mathrm{II}}^{2}}{C_{L}}+\frac{K_{\mathrm{III}}^{2}}{C_{A}}+\frac{K_{\mathrm{I}} K_{\mathrm{D}}}{e}-\frac{K_{\mathrm{D}}^{2}}{\kappa}+\frac{K_{\mathrm{I}} K_{\mathrm{D}}}{e}\right\} .
\end{aligned}
$$

The energy release rate consists of the mechanical terms (for each opening mode) and the electric contribution.

\section{Numerical Examples}

5.1. Electromechanical Griffith Crack (Uniaxial Load). In order to test the accuracy of the CS-XFEM for crack analysis, the methods were applied to a crack in a plane subjected to normal uniaxial tension when $\sigma=1.0 \mathrm{MPa}$ and electrical flux $D=0.001 \mathrm{C} / \mathrm{m}^{2}$. Figure 6 shows the polarization direction as $P$. The distance of the central crack along the $x_{1}$-direction is $2 a$ and side length of the plate is $10 \mathrm{~m}$. At the crack faces, impermeable electric boundary conditions are prescribed. Piezoelectric materials PZT-4, P7, and PZT-H5 were adopted for numerical simulation. The material parameters are shown in Table 1. 
TABLE 1: Material constant.

\begin{tabular}{lcccccccccc}
\hline \multirow{2}{*}{ Material } & \multicolumn{3}{c}{ Elastic constants $/\left(10^{10} \mathrm{~N} / \mathrm{m}^{2}\right)$} & \multicolumn{3}{c}{ Piezoelectric constant $/\left(\mathrm{C} / \mathrm{m}^{2}\right)$} & \multicolumn{3}{c}{ Dielectric constant/(10 $\left.{ }^{10} \mathrm{C} / \mathrm{V} \mathrm{m}\right)$} \\
& $c_{11}$ & $c_{12}$ & $c_{13}$ & $c_{44}$ & $c_{55}$ & $e_{31}$ & $e_{33}$ & $e_{15}$ & $d_{11}$ & $d_{33}$ \\
\hline PZT-4 & 13.9 & 7.78 & 7.43 & 2.56 & 11.3 & -6.98 & 13.84 & 13.44 & 60.0 & 54.7 \\
P-7 & 13.0 & 8.3 & 8.3 & 2.5 & 11.9 & -10.3 & 14.7 & 13.5 & 171.0 & 186.0 \\
PZT-H5 & 12.6 & 7.95 & 8.41 & 2.3 & 11.7 & -6.5 & 23.3 & 17.44 & 150.3 & 130.0 \\
\hline
\end{tabular}

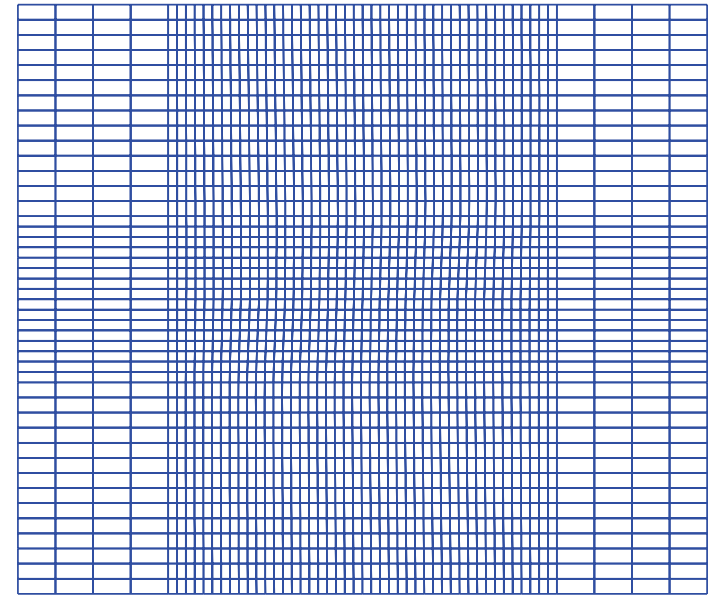

(I) 2288 elements

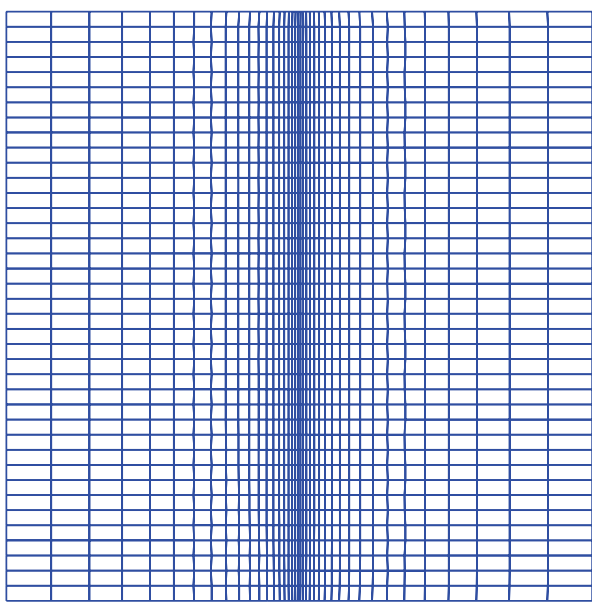

(II) 1521 elements

Figure 7: Mesh of the piezoelectric plate.

The exact analytical solution for the crack in the infinite plane under far-field loads $\sigma_{33}^{\infty}, \sigma_{13}^{\infty}$, and $D_{3}^{\infty}$ was given by Pak [11]. Consider

$$
\begin{aligned}
K_{\mathrm{I}} & =\sigma_{33}^{\infty} \sqrt{\pi a}, \\
K_{\mathrm{II}} & =\sigma_{13}^{\infty} \sqrt{\pi a}, \\
K_{\mathrm{D}} & =D_{3}^{\infty} \sqrt{\pi a} .
\end{aligned}
$$

In order to verify the reliability of CS-XFEM, we set crack length as $2 a=2 \mathrm{~m}$ and use two grid models ((I) 2288 elements, (II) 1521 elements) before the fracture starts, as shown in Figure 7 . There are five types of smoothing elements ((i) split smoothing element: there is one Gauss point on each boundary segment for split smoothing element; (ii) split-blending smoothing element: one Gauss point on each boundary segment is sufficient; (iii) tip smoothing element: five Gauss points on a segment of smoothing element are sufficient; (iv) tip-blending smoothing element: five Gauss points on each boundary segment are sufficient; (v) standard smoothing element: one Gauss point on each boundary segment is sufficient) being used for numerical integration as mentioned in [41]. In the simulation every 4-node calculation grid adopts four smoothing elements. The results are compared with those of the XFEM and the theoretical solutions.

The results of stress intensity factors and electric displacements factors produced by CS-XFEM and XFEM for three loading cases are listed in Table 2. It can be seen that the results of CS-XFEM are closer to the analytical solution than those of XFEM when using the same mesh. This confirms that combining cell-based smoothing technique with the XFEM can improve accuracy.

The normalized mechanical and electrical intensity factors of PZT-4 and P7 with different length of cracks and model (I) grids are listed in Table 3. It is obvious that the CS-XFEM can produce more accurate results than XFEM when using the same number of nodes, which indicates that the smoothing technique adopted in this work improve the calculation of normalized mechanical and electrical intensity factors for fracture in piezoelectric materials.

5.2. Electromechanical Griffith Crack (Shear Load). In the second example of straight electromechanical crack in the plane, the normal mechanical load is replaced by a shear load. The far-field loadings are $\sigma_{33}^{\infty}=0, \sigma_{13}^{\infty}=1 \mathrm{MPa}$, and $D_{3}^{\infty}=0.001 \mathrm{C} / \mathrm{m}^{2}$; see Figure 8 .

From Table 4, we can observe that the result of the mechanical and electrical intensity factors of PZT-H5 has a high precision in two models. The accuracy of CS-XFEM is higher than that of XFEM. The results prove that the CS-XFEM can decrease stiffness of the system and improve solution accuracy. Also model (I) of PZT-H5 was used under the CS-XFEM and XFEM using gauss integral calculation efficiency. CS-XFEM takes 46.247 seconds, while XFEM takes 48.252 second with the following CPU setup: Intel Core i5$34703.20 \mathrm{GHz}$, RAM: $8 \mathrm{G}$. The efficiency of CS-XFEM has been improved but is not obvious.

The normalized mechanical and electrical intensity factors of PZT-4 and P7 with different length of cracks when using model (I) grids are listed in Table 5. It is obvious that 


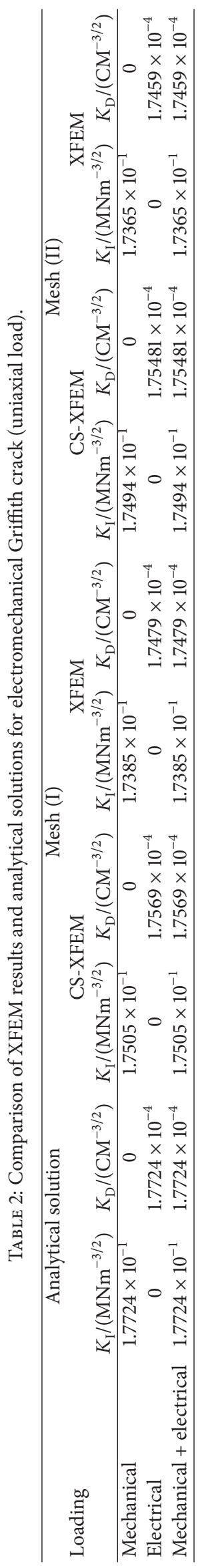


TABLE 3: Normalized intensity factors under different crack lengths for electromechanical Griffith crack (uniaxial load).

\begin{tabular}{|c|c|c|c|c|c|}
\hline \multirow{2}{*}{$a$} & \multirow{2}{*}{ Normalized intensity factors } & \multicolumn{2}{|c|}{ PZT-4 } & \multicolumn{2}{|c|}{ P-7 } \\
\hline & & CS-XFEM & XFEM & CS-XFEM & XFEM \\
\hline \multirow{2}{*}{$0.5 \mathrm{~m}$} & $K_{\mathrm{I}} / \sigma_{33}^{\infty} \sqrt{\pi a}$ & 0.989 & 0.965 & 0.978 & 0.967 \\
\hline & $K_{\mathrm{D}} / D_{33}^{\infty} \sqrt{\pi a}$ & 0.994 & 0.974 & 0.986 & 0.978 \\
\hline \multirow{2}{*}{$1.0 \mathrm{~m}$} & $K_{\mathrm{I}} / \sigma_{33}^{\infty} \sqrt{\pi a}$ & 0.941 & 0.932 & 0.948 & 0.925 \\
\hline & $K_{\mathrm{D}} / D_{33}^{\infty} \sqrt{\pi a}$ & 0.963 & 0.940 & 0.956 & 0.948 \\
\hline \multirow{2}{*}{$1.5 \mathrm{~m}$} & $K_{\mathrm{I}} / \sigma_{33}^{\infty} \sqrt{\pi a}$ & 0.983 & 0.966 & 0.988 & 0.978 \\
\hline & $K_{\mathrm{D}} / D_{33}^{\infty} \sqrt{\pi a}$ & 0.987 & 0.973 & 0.979 & 0.979 \\
\hline \multirow{2}{*}{$2.0 \mathrm{~m}$} & $K_{\mathrm{I}} / \sigma_{33}^{\infty} \sqrt{\pi a}$ & 0.991 & 0.969 & 0.993 & 0.970 \\
\hline & $K_{\mathrm{D}} / D_{33}^{\infty} \sqrt{\pi a}$ & 0.993 & 0.987 & 0.984 & 0.978 \\
\hline
\end{tabular}

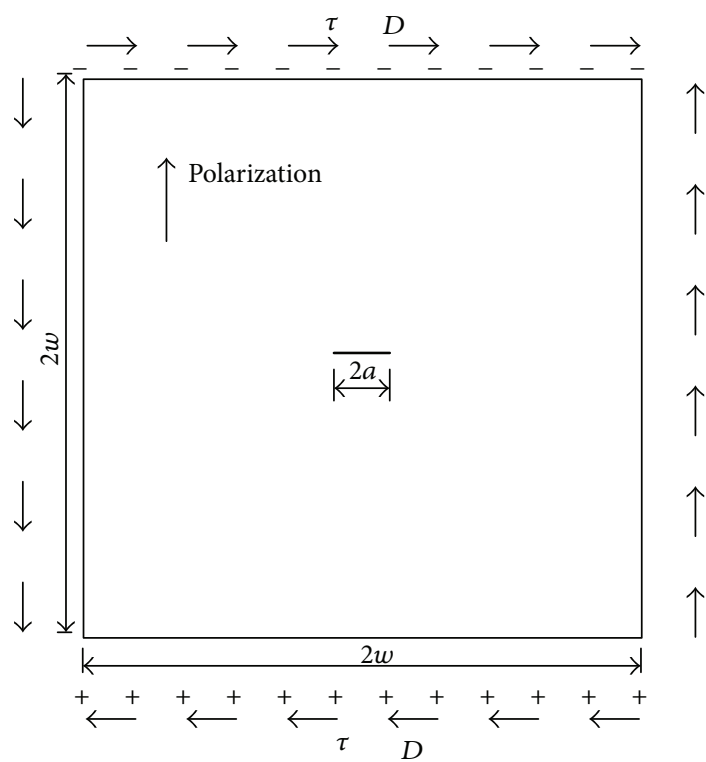

FIGURE 8: Electromechanical Griffith crack (uniaxial load).

the CS-XFEM can produce more accurate results than XFEM using the same number of nodes.

5.3. Piezoelectric Model with a Hole with Cracks. A piezoelectric model, with a center circular hole $R=10 \mathrm{~mm}$ and horizontal cracks $a$ on the left and right, is subjected to unidirectional uniform tensile $\sigma^{\infty}=10 \mathrm{MPa}$ and electric displacement $D^{\infty}=10^{-3} \mathrm{C} / \mathrm{m}^{2}$ at infinity. The geometric model is expressed as in Figure 9, the length of side $l=$ $200 \mathrm{~mm}, P$ is the direction of polarization, and material is PZT-H5. The situation of meshing when $a=4 \mathrm{~mm}$ was given by Figure 10 .

The stress intensity factor and electric displacement intensity factor on the left side of the crack tip obtained by CS-XFEM and XFEM in different crack lengths are shown in Table 6. The maximum error of calculation in CS-XFEM is $2.9 \%$, and the maximum error of calculation in XFEM is 4.1\%. The results verified the accuracy of CS-XFEM.

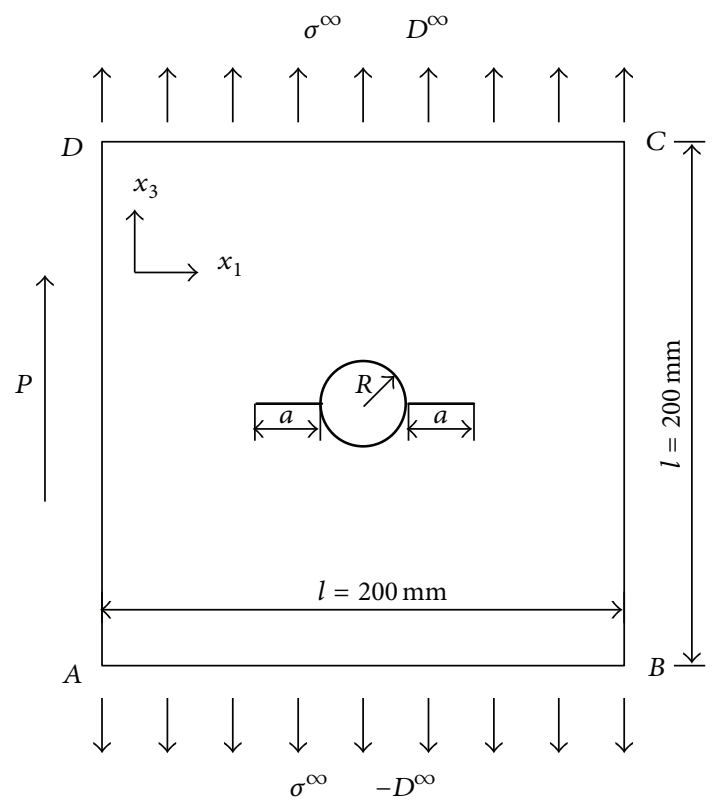

Figure 9: Piezoelectric model with a hole with cracks.

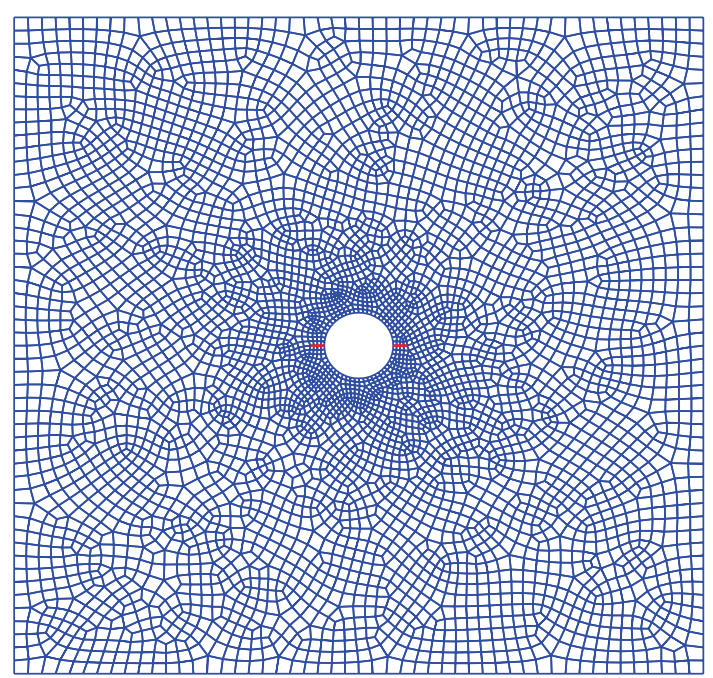

Figure 10: Discrete model. 


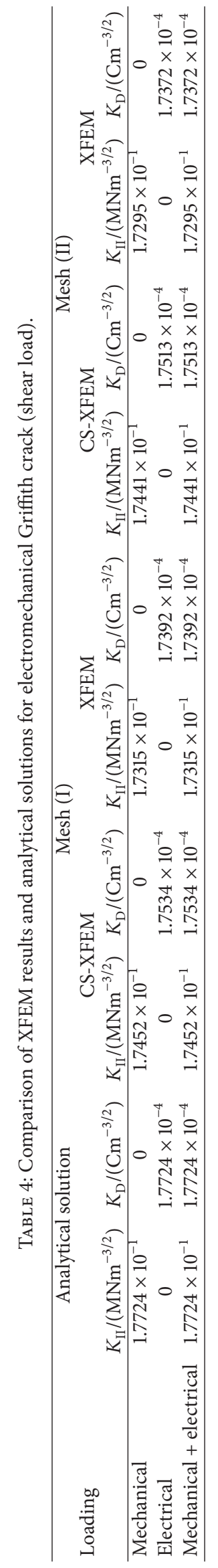


TABLE 5: Normalized intensity factors under different crack lengths for electromechanical Griffith crack (shear load).

\begin{tabular}{|c|c|c|c|c|c|}
\hline \multirow{2}{*}{$a$} & \multirow{2}{*}{ Normalized intensity factors } & \multicolumn{2}{|c|}{ PZT-4 } & \multicolumn{2}{|c|}{ P-7 } \\
\hline & & CS-XFEM & XFEM & CS-XFEM & XFEM \\
\hline \multirow{2}{*}{$0.5 \mathrm{~m}$} & $K_{\mathrm{II}} / \tau_{13}^{\infty} \sqrt{\pi a}$ & 0.988 & 0.979 & 0.977 & 0.964 \\
\hline & $K_{\mathrm{D}} / D_{33}^{\infty} \sqrt{\pi a}$ & 0.994 & 0.972 & 0.985 & 0.971 \\
\hline \multirow{2}{*}{$1.0 \mathrm{~m}$} & $K_{\mathrm{II}} / \tau_{13}^{\infty} \sqrt{\pi a}$ & 0.968 & 0.948 & 0.957 & 0.937 \\
\hline & $K_{\mathrm{D}} / D_{33}^{\infty} \sqrt{\pi a}$ & 0.986 & 0.961 & 0.975 & 0.958 \\
\hline \multirow{2}{*}{$1.5 \mathrm{~m}$} & $K_{\mathrm{II}} / \tau_{13}^{\infty} \sqrt{\pi a}$ & 0.958 & 0.940 & 0.948 & 0.935 \\
\hline & $K_{\mathrm{D}} / D_{33}^{\infty} \sqrt{\pi a}$ & 0.973 & 0.955 & 0.963 & 0.940 \\
\hline \multirow{2}{*}{$2.0 \mathrm{~m}$} & $K_{\mathrm{II}} / \tau_{13}^{\infty} \sqrt{\pi a}$ & 0.994 & 0.987 & 0.981 & 0.972 \\
\hline & $K_{\mathrm{D}} / D_{33}^{\infty} \sqrt{\pi a}$ & 0.998 & 0.991 & 0.996 & 0.989 \\
\hline
\end{tabular}

TABLE 6: Intensity factor under different crack lengths.

\begin{tabular}{lcccc}
\hline$a$ & Intensity factor & CS-XFEM & XFEM & $\begin{array}{c}\text { Analytical } \\
\text { solution }\end{array}$ \\
\hline \multirow{2}{*}{$1 \mathrm{~mm}$} & $K_{\mathrm{I}} / 10^{7} \times \mathrm{Pa} \cdot \mathrm{mm}^{0.5}$ & 0.2995 & 0.2950 & 0.3076 \\
& $K_{\mathrm{D}} / 10^{-9} \times \mathrm{C} \cdot \mathrm{mm}^{-1.5}$ & 0.2986 & 0.2983 & 0.3076 \\
\hline \multirow{2}{*}{$2 \mathrm{~mm}$} & $K_{\mathrm{I}} / 10^{7} \times \mathrm{Pa} \cdot \mathrm{mm}^{0.5}$ & 0.5322 & 0.5263 & 0.5416 \\
& $K_{\mathrm{D}} / 10^{-9} \times \mathrm{C} \cdot \mathrm{mm}^{-1.5}$ & 0.5300 & 0.5230 & 0.5416 \\
\hline \multirow{2}{*}{$4 \mathrm{~mm}$} & $K_{\mathrm{I}} / 10^{7} \times \mathrm{Pa} \cdot \mathrm{mm}^{0.5}$ & 0.8513 & 0.8368 & 0.8681 \\
& $K_{\mathrm{D}} / 10^{-9} \times \mathrm{C} \cdot \mathrm{mm}^{-1.5}$ & 0.8454 & 0.8351 & 0.8681 \\
\hline \multirow{2}{*}{$6 \mathrm{~mm}$} & $K_{\mathrm{I}} / 10^{7} \times \mathrm{Pa} \cdot \mathrm{mm}^{0.5}$ & 1.0517 & 1.0560 & 1.0800 \\
& $K_{\mathrm{D}} / 10^{-9} \times \mathrm{C} \cdot \mathrm{mm}^{-1.5}$ & 1.0603 & 1.0432 & 1.0800 \\
\hline
\end{tabular}

\section{Conclusions}

With the growing applications of piezoelectric structures in innovative technical areas, problems of strength and reliability become important and have to be carefully investigated. In order to quantitatively assess fracture and fatigue, sophisticated analysis of cracks' electromechanical properties is needed. The fracture mechanics approach for crack-like defects in piezoelectric materials reveals coupled electrical and mechanical field singularities. Effective numerical methods are needed to evaluate fracture behavior of cracks in arbitrary piezoelectric structures subjected to combined electromechanical loading.

In this paper CS-XFEM with crack tip enrichment functions was presented for electromechanical crack analyses. Two examples are used to verify the accuracy of CS-XFEM. Through the numerical simulation conclusions can be drawn as follows:

(1) The cell-based smoothing technique is extended into XFEM to combine the advantages of XFEM and CS-FEM. Thus, CS-XFEM has high accuracy and convergence rate. The present method also simplifies the integration of discontinuous approximation by transforming interior integration into boundary integration. More importantly, no derivatives of shape functions are needed to compute the stiffness matrix.

(2) The examples show that CS-XFEM can perform better in accuracy and convergence rate compared with
XFEM in terms of stress intensity factors and electric displacements factors.

(3) CS-XFEM is superior to XFEM in regard to the calculation of the stiffness matrix and singularity in the integrand. It also avoids the mapping process, which will increase the complexity of the calculation.

\section{Conflict of Interests}

The authors declare that there is no conflict of interests regarding the publication of this paper.

\section{Acknowledgments}

This work was financially supported by the National Natural Science Foundation of China (Grant no. 51305157), the National Key Scientific Instrument and Equipment Development Projects, China (Grant no. 2012YQ030075), and Jilin Provincial Department of Science and Technology Fund Project (Grant no. 20130305006GX).

\section{References}

[1] M. Li, J. X. Yuan, D. Guan, and W. M. Chen, "Application of piezoelectric fiber composite actuator to aircraft wing for aerodynamic performance improvement," Science China Technological Sciences, vol. 54, no. 2, pp. 395-402, 2011.

[2] M. Kuna, "Finite element analyses of crack problems in piezoelectric structures," Computational Materials Science, vol. 13, no. 1-3, pp. 67-80, 1998.

[3] J. Meckerle, "Smart materials and structures-a finite element approach-an addendum: a bibliography (1997-2002)," Modelling and Simulation in Materials Science and Engineering, vol. 11, no. 5, pp. 707-744, 2003.

[4] F. García-Sánchez, Ch. Zhang, and A. Sáez, “2-D transient dynamic analysis of cracked piezoelectric solids by a timedomain BEM," Computer Methods in Applied Mechanics and Engineering, vol. 197, no. 33-40, pp. 3108-3121, 2008.

[5] Ch. Zhang, F. García-Sánchez, and A. Sáez, “Time-domain BEM analysis of cracked piezoelectric solids under impact loading," in Computational Mechanics: Proceedings of "International Symposium on Computational Mechanics" July 30-August 1, 2007, Beijing, China, pp. 206-218, Springer, Berlin, Germany, 2009. 
[6] J. Sladek, V. Sladek, C. Zhang, P. Solek, and L. Starek, "Fracture analyses in continuously nonhomogeneous piezoelectric solids by the MLPG," Computer Modeling in Engineering and Sciences, vol. 19, no. 3, pp. 247-262, 2007.

[7] S. Nanthakumar, T. Lahmer, X. Zhuang, G. Zi, and T. Rabczuk, "Detection of material interfaces using a regularized level set method in piezoelectric structures," Inverse Problems in Science and Engineering, pp. 1-24, 2015.

[8] S. S. Nanthakumar, T. Lahmer, and T. Rabczuk, "Detection of flaws in piezoelectric structures using extended FEM," International Journal for Numerical Methods in Engineering, vol. 96, no. 6, pp. 373-389, 2013.

[9] S. S. Nanthakumar, T. Lahmer, and T. Rabczuk, "Detection of multiple flaws in piezoelectric structures using XFEM and level sets," Computer Methods in Applied Mechanics and Engineering, vol. 275, pp. 98-112, 2014.

[10] Z. Suo, C.-M. Kuo, D. M. Barnett, and J. R. Willis, "Fracture mechanics for piezoelectric ceramics," Journal of the Mechanics and Physics of Solids, vol. 40, no. 4, pp. 739-765, 1992.

[11] Y. E. Pak, "Linear electro-elastic fracture mechanics of piezoelectric materials," International Journal of Fracture, vol. 54, no. 1, pp. 79-100, 1992.

[12] X.-L. Xu and R. K. N. D. Rajapakse, "Analytical solution for an arbitrarily oriented void/crack and fracture of piezoceramics," Acta Materialia, vol. 47, no. 6, pp. 1735-1747, 1999.

[13] H. Sosa, "Plane problems in piezoelectric media with defects," International Journal of Solids and Structures, vol. 28, no. 4, pp. 491-505, 1991.

[14] M. Kuna, "Fracture mechanics of piezoelectric materialswhere are we right now?" Engineering Fracture Mechanics, vol. 77, no. 2, pp. 309-326, 2010.

[15] M. Kuna, "Finite element analyses of cracks in piezoelectric structures: a survey," Archive of Applied Mechanics, vol. 76, no. 11-12, pp. 725-745, 2006.

[16] R. R. Bhargava and K. Sharma, "X-FEM studies on an inclined crack in a 2-D finite piezoelectric media," in Future Communication, Computing, Control and Management, vol. 141 of Lecture Notes in Electrical Engineering, pp. 285-290, Springer, Berlin, Germany, 2012.

[17] M. Abendroth, U. Groh, M. Kuna, and A. Ricoeur, "Finite element-computation of the electromechanical J-integral for 2$\mathrm{D}$ and 3-D crack analysis," International Journal of Fracture, vol. 114, no. 4, pp. 359-378, 2002.

[18] Y. Motola and L. Banks-Sills, "M-integral for calculating intensity factors of cracked piezoelectric materials using the exact boundary conditions," Journal of Applied Mechanics, vol. 76, no. 1, Article ID 011004, 9 pages, 2009.

[19] R. R. Bhargava and K. Sharma, "A study of finite size effects on cracked 2-D piezoelectric media using extended finite element method," Computational Materials Science, vol. 50, no. 6, pp. 1834-1845, 2011.

[20] H. Nguyen-Vinh, I. Bakar, M. A. Msekh et al., "Extended finite element method for dynamic fracture of piezo-electric materials," Engineering Fracture Mechanics, vol. 92, pp. 19-31, 2012.

[21] G. R. Liu, T. T. Nguyen, K. Y. Dai, and K. Y. Lam, “Theoretical aspects of the smoothed finite element method (SFEM)," International Journal for Numerical Methods in Engineering, vol. 71, no. 8, pp. 902-930, 2007.

[22] T. Nguyen-Thoi, T. Bui-Xuan, P. Phung-Van, H. Nguyen-Xuan, and P. Ngo-Thanh, "Static, free vibration and buckling analyses of stiffened plates by CS-FEM-DSG3 using triangular elements," Computers \& Structures, vol. 125, pp. 100-113, 2013.

[23] C. V. Le, H. Nguyen-Xuan, H. Askes et al., "A cell-based smoothed finite element method for kinematic limit analysis," International Journal for Numerical Methods in Engineering, vol. 83, no. 12, pp. 1651-1674, 2010.

[24] G. R. Liu, T. Nguyen-Thoi, and K. Y. Lam, "An edge-based smoothed finite element method (ES-FEM) for static, free and forced vibration analyses of solids," Journal of Sound and Vibration, vol. 320, no. 4-5, pp. 1100-1130, 2009.

[25] N. Nguyen-Thanh, T. Rabczuk, H. Nguyen-Xuan, and S. P. A. Bordas, "An alternative alpha finite element method (A $\alpha$ FEM) for free and forced structural vibration using triangular meshes," Journal of Computational and Applied Mathematics, vol. 233, no. 9, pp. 2112-2135, 2010.

[26] T. Nguyen-Thoi, G. R. Liu, K. Y. Lam, and G. Y. Zhang, "A facebased smoothed finite element method (FS-FEM) for 3D linear and geometrically non-linear solid mechanics problems using 4-node tetrahedral elements," International Journal for Numerical Methods in Engineering, vol. 78, no. 3, pp. 324-353, 2009.

[27] H. Nguyen-Xuan, T. Rabczuk, N. Nguyen-Thanh, T. NguyenThoi, and S. Bordas, "A node-based smoothed finite element method with stabilized discrete shear gap technique for analysis of Reissner-Mindlin plates," Computational Mechanics, vol. 46, no. 5, pp. 679-701, 2010.

[28] H. Nguyen-Xuan, L. V. Tran, T. Nguyen-Thoi, and H. C. VuDo, "Analysis of functionally graded plates using an edge-based smoothed finite element method," Composite Structures, vol. 93, no. 11, pp. 3019-3039, 2011.

[29] G. R. Liu, H. Nguyen-Xuan, T. Nguyen-Thoi, and X. Xu, "A novel Galerkin-like weakform and a superconvergent alpha finite element method ( $\alpha$ FEM) for mechanics problems using triangular meshes," Journal of Computational Physics, vol. 228, no. 11, pp. 4055-4087, 2009.

[30] H. Nguyen-Xuan, L. V. Tran, C. H. Thai, and T. Nguyen-Thoi, "Analysis of functionally graded plates by an efficient finite element method with node-based strain smoothing," ThinWalled Structures, vol. 54, pp. 1-18, 2012.

[31] H. Nguyen-Xuan, T. Rabczuk, T. Nguyen-Thoi, T. N. Tran, and N. Nguyen-Thanh, "Computation of limit and shakedown loads using a node-based smoothed finite element method," International Journal for Numerical Methods in Engineering, vol. 90, no. 3, pp. 287-310, 2012.

[32] H. Nguyen-Xuan, G. R. Liu, N. Nourbakhshnia, and L. Chen, "A novel singular ES-FEM for crack growth simulation," Engineering Fracture Mechanics, vol. 84, pp. 41-66, 2012.

[33] N. Nourbakhshnia and G. R. Liu, "A quasi-static crack growth simulation based on the singular ES-FEM," International Journal for Numerical Methods in Engineering, vol. 88, no. 5, pp. 473492, 2011.

[34] W. Zeng, G. R. Liu, Y. Kitamura, and H. Nguyen-Xuan, "A threedimensional ES-FEM for fracture mechanics problems in elastic solids," Engineering Fracture Mechanics, vol. 114, pp. 127-150, 2013.

[35] P. Phung-Van, T. Nguyen-Thoi, T. Le-Dinh, and H. NguyenXuan, "Static and free vibration analyses and dynamic control of composite plates integrated with piezoelectric sensors and actuators by the cell-based smoothed discrete shear gap method (CS-FEM-DSG3)," Smart Materials and Structures, vol. 22, no. 9, Article ID 095026, 2013. 
[36] P. Phung-Van, L. De Lorenzis, C. H. Thai, M. Abdel-Wahab, and H. Nguyen-Xuan, "Analysis of laminated composite plates integrated with piezoelectric sensors and actuators using higherorder shear deformation theory and isogeometric finite elements," Computational Materials Science, vol. 96, pp. 495-505, 2015.

[37] S. P. A. Bordas, T. Rabczuk, N.-X. Hung et al., "Strain smoothing in FEM and XFEM," Computers \& Structures, vol. 88, no. 23-24, pp. 1419-1443, 2010.

[38] X. Zhao, S. P. A. Bordas, and J. Qu, "A hybrid smoothed extended finite element/level set method for modeling equilibrium shapes of nano-inhomogeneities," Computational Mechanics, vol. 52, no. 6, pp. 1417-1428, 2013.

[39] L. Chen, T. Rabczuk, S. P. A. Bordas, G. R. Liu, K. Y. Zeng, and P. Kerfriden, "Extended finite element method with edge-based strain smoothing (ESm-XFEM) for linear elastic crack growth," Computer Methods in Applied Mechanics and Engineering, vol. 209-212, pp. 250-265, 2012.

[40] Y. Jiang, T. E. Tay, L. Chen, and X. S. Sun, "An edge-based smoothed XFEM for fracture in composite materials," International Journal of Fracture, vol. 179, no. 1-2, pp. 179-199, 2013.

[41] N. Vu-Bac, H. Nguyen-Xuan, L. Chen et al., "A node-based smoothed extended finite element method (NS-XFEM) for fracture analysis," Computer Modeling in Engineering and Sciences, vol. 73, no. 4, pp. 331-355, 2011.

[42] E. Bechet, M. Scherzer, and M. Kuna, "Application of the X-FEM to the fracture of piezoelectric materials," International Journal for Numerical Methods in Engineering, vol. 77, pp. 1535-1599, 2009.

[43] U. Groh and M. Kuna, "Efficient boundary element analysis of cracks in 2D piezoelectric structures," International Journal of Solids and Structures, vol. 42, no. 8, pp. 2399-2416, 2005.

[44] L. M. Zhou, G. W. Meng, F. Li, and H. Wang, "Cell-based smoothed finite element method-virtual crack closure technique for a piezoelectric material of crack," Mathematical Problems in Engineering, vol. 2015, Article ID 371083, 10 pages, 2015.

[45] P. Laborde, J. Pommier, Y. Renard, and M. Salaün, "High-order extended finite element method for cracked domains," International Journal for Numerical Methods in Engineering, vol. 64, no. 3, pp. 354-381, 2005.

[46] N. Moës, J. Dolbow, and T. Belytschko, "A finite element method for crack growth without remeshing," International Journal for Numerical Methods in Engineering, vol. 46, no. 1, pp. 131-150, 1999.

[47] T. Rabczuk and T. Belytschko, "Cracking particles: a simplified mesh-free method for arbitrary evolving cracks," International Journal for Numerical Methods in Engineering, vol. 61, no. 13, pp. 2316-2343, 2004.

[48] T. Rabczuk, G. Zi, S. Bordas, and H. Nguyen-Xuan, "A geometrically non-linear three-dimensional cohesive crack method for reinforced concrete structures," Engineering Fracture Mechanics, vol. 75, no. 16, pp. 4740-4758, 2008.

[49] J. R. Rice, "Mathematical analysis in the mechanics of fracture," in Fracture: An Advanced Treatise, H. Liebowitz, Ed., vol. 2, pp. 191-311, Academic Press, New York, NY, USA, 1968.

[50] J. D. Eshelby, "Energy relations and the energy-momentum tensor in continuum mechanics," in Inelastic Behavior of Solids, M. F. Kanninen, W. F. Adler, A. R. Rosenfield, and R. I. Jaffee, Eds., pp. 77-115, McGraw-Hill, New York, NY, USA, 1970. 

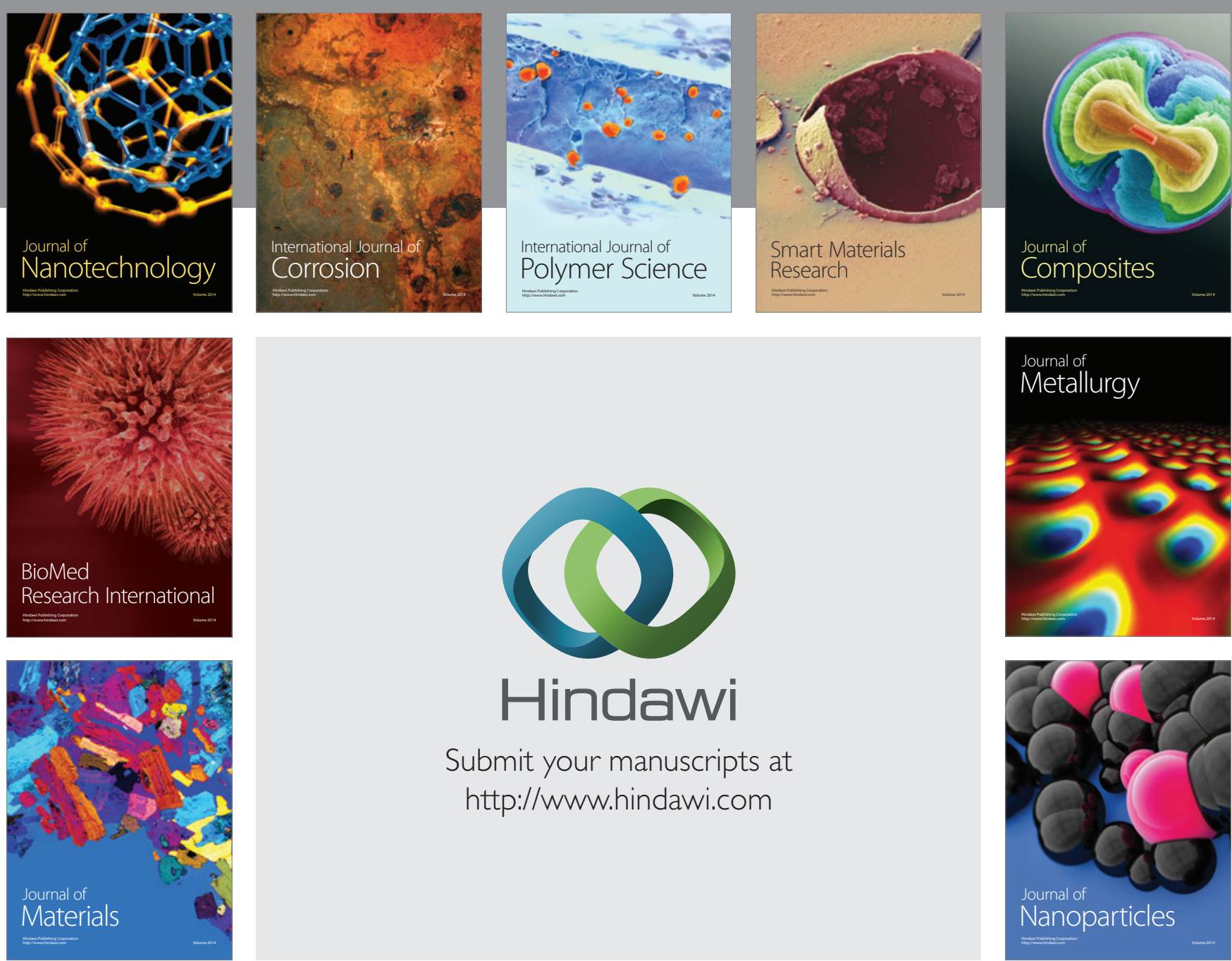

\section{Hindawi}

Submit your manuscripts at

http://www.hindawi.com

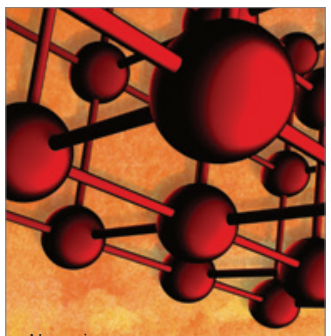

Materials Science and Engineering
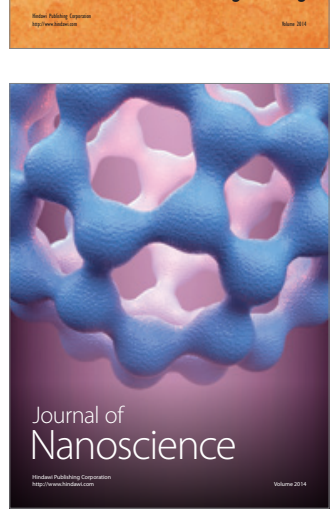
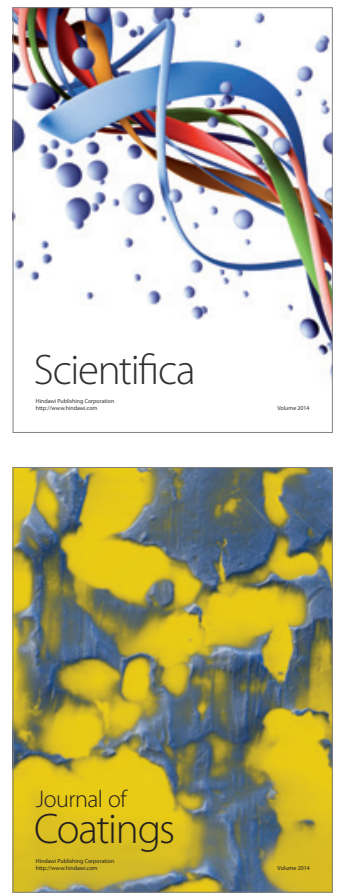
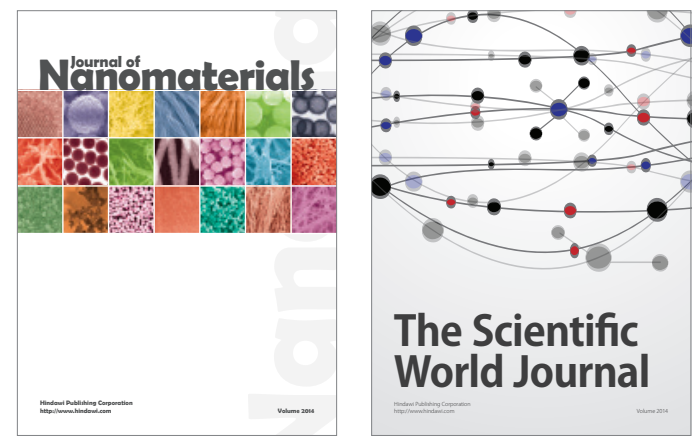

The Scientific World Journal
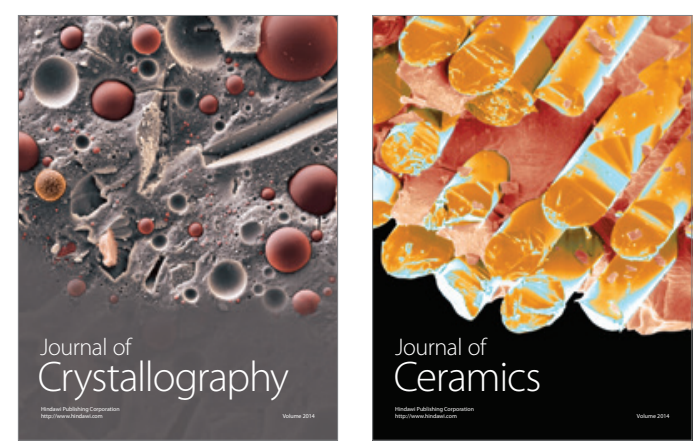
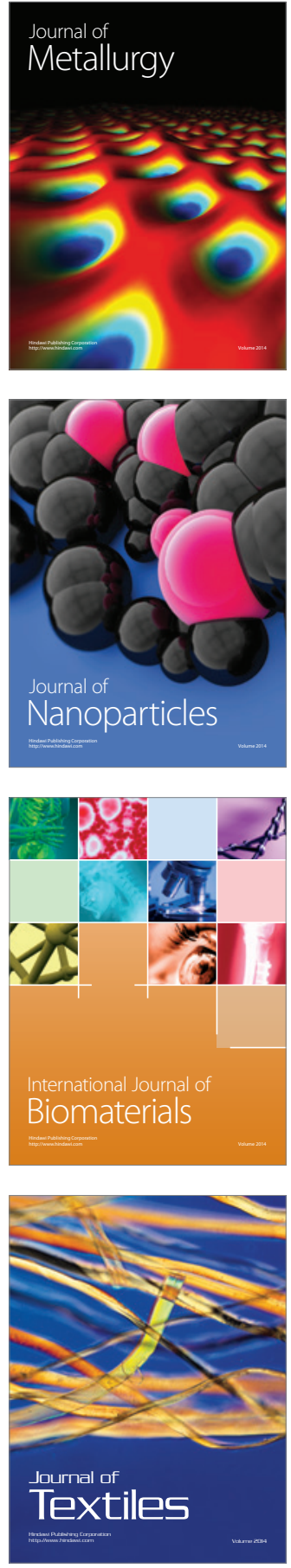